\title{
Efetividade de um programa de exercícios no condicionamento físico, perfil metabólico e pressão arterial de pacientes hipertensos
}

\author{
Henrique L. Monteiro, PhD¹, Lívia M.C. Rolim¹, Daniela A. Squinca', Fernando C. Silva1, \\ Carla C.C. Ticianeli, MD² e Sandra L. Amaral, PhD'1
}

\section{RESUMO}

Histórico e objetivo: Tem sido mostrado que exercícios aeróbios são úteis na redução da pressão arterial. Contudo, a efetividade de um programa de exercícios ainda é controversa e não foi bem analisada em populações de baixa renda. O objetivo do presente estudo foi estabelecer um programa de condicionamento físico individualizado - Projeto Hipertensão - focado em pessoas hipertensas, pacientes da Unidade Básica de Saúde (UBS) e, logo após, investigar os efeitos deste programa no condicionamento físico, perfil metabólico e níveis de pressão. Métodos: Dezesseis mulheres hipertensas (56 \pm 3 anos) sob tratamento farmacológico regular foram submetidas a 4 meses de um programa de exercícios aeróbios e de alongamento ( 3 sessões/semana, $90 \mathrm{~min} / \mathrm{ses}-$ são, $60 \%$ de $\mathrm{VO}_{2}$ max.) Diversas variáveis físicas e metabólicas foram comparadas antes e depois de 4 meses de treinamento. Resultados: $O$ treinamento diminuiu significativamente a pressão arterial sistólica (PAS, $-6 \%$ ); melhorou o condicionamento cardiorrespiratório $\left(+42 \%\right.$ do $\dot{\mathrm{V}}_{2}$ max), flexibilidade $(+11 \%)$ e conteúdo de glicose plasmática ( $-4 \%)$. IMC e \% de gordura não tiveram modificação. Além de modificar o perfil metabólico, observou-se que o treinamento apresentou correlações significativas entre os valores iniciais individuais de nível de colesterol total (CT), lipoproteína de alta densidade (HDL-C) e lipoproteína de baixa densidade $(L D L-C)$ e suas respostas após exercício. Conclusões: $O$ estudo mostra que programas de exercício podem ser personalizados para pacientes hipertensos da UBS e confirma a efetividade do exercício na PA, condicionamento físico, flexibilidade e perfil lipídico em pacientes hipertensos. A redução expressiva de PA em sujeitos hipertensos sugere que esta intervenção de exercícios deve ser enfatizada em outros centros que assistam populações de baixa renda.

\section{ABSTRACT \\ Exercise program effectiveness on physical fitness, metabolic profile and blood pressure of hypertensive patients}

Background and objective: It has been shown that aerobic exercise is useful to reduce arterial pressure, however, the effectiveness of an exercise program is still controversial and not very well analyzed among populations with low-income. The objective

1. Laboratório de Avaliação e Prescrição de Exercícios (LAPE/UNESP), Departamento de Educação Física, Faculdade de Ciências, Universidade Estadual Paulista - Unesp, Bauru, SP, Brasil.

2. Núcleo de Saúde Otávio Rasi, Bauru, SP, Brasil.

Recebido em 26/1/06. Versão final recebida em 31/8/06. Aceito em 13/10/06.

Endereço para correspondência: Sandra Lia do Amaral, PhD, Department of Physical Education, Science Faculty, UNESP - São Paulo State University - Bauru, SP, Brasil. Tel.: 55-14-3103-6082, fax: 55-14-3103-6071.

E-mail: slamaral@fc.unesp.br
Palavras-chave: Pressão arterial. Treinamento físico. Perfil lipídico.

Keywords: Arterial pressure. Exercise training. Lipid profile.

of the present study was to set up an individualized physical fitness program - Projeto Hipertensão - focused on hypertensive people, patients from a Health Basic Unit (HBU) and, after that, to investigate the effects of this program on physical fitness, metabolic profile and pressure levels. Methods: Sixteen hypertensive women (56 $\pm 3 y$ rs) under regular pharmacological treatment underwent 4 months of a supervised aerobic and stretching exercise program (3 sessions/wk, $90 \mathrm{~min} /$ session, $60 \%$ of $\dot{\mathrm{VO}}_{2}$ max). Several physical and metabolic variables were compared before and after 4 months of training. Results: Training significantly reduced systolic arterial pressure (SAP, -6\%), improved cardiorespiratory fitness $\left(+42 \%\right.$ of $\dot{V}_{2}$ max), flexibility $(+11 \%)$ and plasma glucose content (-4\%). BMI and \% fat did not change. Besides modifying metabolic profile, it was found that training presented significant correlations between individual initial values of cholesterol total level (CT), high density lipoprotein (HDL-C) and low density lipoprotein ( $L D L-C)$ and its responses after exercise. Conclusions: The study shows that exercise programs can be personalized for hypertensive patients from a HBU and confirms the effectiveness of exercise on AP, physical fitness, flexibility and lipid profile on hypertensive patients. The expressive reduction of AP in hypertensive subjects suggests that this exercise intervention should be emphasized on other health centers which assist low-income population.

\section{INTRODUÇÃO}

A pressão arterial alta (PA) é um fator de risco poderoso, independente e o mais importante para doenças cardiovasculares (DCV), além disso, se tornou a maior doença endêmica mundial(14). Segundo o 70 Comitê Nacional em Prevenção, Detecção, Avaliação e Tratamento da Pressão Arterial Alta(4), indivíduos com PA sistólica (PAS) de 120 a 139mmHg ou PA diastólica (PAD) de 80 a $89 \mathrm{mmHg}$ devem ser identificados como pré-hipertensos e requerem modificações que promovam saúde no estilo de vida para prevenir a progressão para hipertensão e $\mathrm{DCV} V^{(4)}$. Quando não tratada, a hipertensão pode causar sérios problemas como doença coronária, enfarte, doença congestiva do coração, doença renal, doença vascular periférica e doenças cerebrais, contribuindo assim para o aumento da taxa de morbidade e mortalidade no mundo inteiro(1). Aproximadamente $20-30 \%$ dos brasileiros têm PA acima do nível recomendado(5), e suas conseqüências são responsáveis por $40 \%$ das aposentadorias precoces ${ }^{(6)}$. Por esta razão, modificações no estilo de vida, incluindo alimentação e exercício físico, são as primeiras linhas de intervenção para o controle de PA alta, mesmo quando terapia medicamentosa é implementada. 
Estudos epidemiológicos têm mostrado uma correlação negativa entre atividade física e hipertensão, significando que pessoas ativas têm menos chances de se tornarem hipertensas. De acordo com tal tese, Church et al. ${ }^{(7)}$ analisando os resultados de 22.167 homens num acompanhamento de 23 anos, demonstraram que a taxa de mortalidade era maior entre aqueles com menor capacidade física. Mais recentemente, Mokdad et al. ${ }^{(8)}$ demonstraram que alimentação ruim e inatividade física eram responsáveis por 16,6\% dos casos atuais de morte nos Estados Unidos, atingindo aproximadamente cerca de 15.000 mortes/ano. No Brasil, os números não são diferentes: o Sistema Nacional de Saúde, entre os anos de 2000-2001, mostrou que das 1.800.155 internações hospitalares por DCV, 14,95\% eram decorrentes de hipertensão. Para outras causas de internações hospitalares, $80 \%$ eram relacionadas a níveis altos de pressão arterial. Alta morbidade, mortalidade e custos elevados associados à drogas, patologia, radiologia e complicações devido a enfartes, doença coronária, doença renal, falha cardíaca e doença renal em estágio final, fazem da hipertensão um importante fator de risco médico modificável. Aproximadamente $20-30 \%$ da população brasileira adulta tem PA acima dos níveis recomendados. Nossa população envelhecerá em alguns anos e este dado estatístico aumenta na medida em que a população envelhece, o que reforça a importância de se encontrar um tratamento eficiente para a hipertensão(5).

Tem sido demonstrado que o exercício físico é eficiente na redução da pressão arterial, porém, a quantidade, o tipo e intensidade do exercício ainda são controversos. Apesar de não haver dúvidas relacionadas aos benefícios do treinamento físico no controle da pressão arterial e respostas metabólicas de pessoas hipertensas, há poucos estudos que avaliaram os resultados, em longo, prazo de um programa de exercícios físicos em pacientes hipertensos de baixa renda provenientes de Unidade Básica de Saúde. A maioria deles são grupos bem organizados, com várias situações ambientais bem controladas e técnicas de alto custo. Por esta razão, o Departamento de Educação Física da UNESP, Bauru, decidiu criar um programa de condicionamento físico individualizado direcionado a pacientes hipertensos de uma Unidade Básica de Saúde. O objetivo do presente estudo foi então estabelecer o "Projeto Hipertensão" na Unidade Básica de Saúde Otávio Rasi, localizada na cidade de Bauru, SP e depois investigar os efeitos deste programa de exercício físico no condicionamento físico, perfil metabólico e níveis de pressão arterial.

\section{MÉTODOS}

\section{Seleção dos pacientes}

Os sujeitos inscritos neste estudo foram recrutados do Centro de Saúde Otavio Rasi, em Bauru, São Paulo. Dentre os 298 pacientes deste centro de saúde, 50 com hipertensão leve a moderada foram escolhidos para participar de um treinamento físico de longa duração em colaboração com o Departamento de Educação Física da Faculdade de Ciências - UNESP - Universidade Estadual Paulista - Bauru. Ao fim do programa, um total de 40 pessoas com hipertensão essencial leve a moderada (8 homens; 32 muIheres) foram inscritos neste programa de 16 semanas. Todos os pacientes estavam sob tratamento farmacológico regular para hipertensão, o qual incluía beta-bloqueadores, diuréticos, inibidores da enzima de conversão da angiotensina II, antagonistas dos canais de cálcio e vasodilatadores. Nenhum paciente apresentou contra-indicação para exercício. Um consentimento esclarecido foi obtido dos pacientes e o estudo foi aprovado pelo Comitê de Ética do da Secretaria de Saúde.

Todos os pacientes foram submetidos à avaliação cardíaca anterior ao treinamento físico, bem como a exames laboratoriais, os quais incluíram dosagens de colesterol total (CT), lipoproteína de alta densidade (HDL-C), lipoproteína de baixa densidade (LDL-C), triglicerídeos (TG) e níveis plasmáticos de glicose. Todos os exames laboratoriais foram repetidos após 4 meses no Laboratório de Bauru. Após consentimento médico, os pacientes foram encaminhados ao Departamento de Educação Física, UNESP, onde foram submetidos a diferentes testes para avaliação da capacidade física e cardiorrespiratórias. A pressão arterial foi medida com um esfigmomanômetro com aneróide 3 vezes após um repouso sentado de 10 minutos com o braço na posição da altura do coração. Uma média dessas medidas foi considerada como pressão arterial de repouso. Estas medidas foram coletadas por monitores treinados (cada monitor era responsável por 5 pacientes para evitar diferenças entre os avaliadores). O esfigmomanômetro foi previamente calibrado contra a coluna de mercúrio e adaptado ao tamanho do braço do paciente, de acordo com as $V$ Diretrizes Brasileiras de Hipertensão Arterial(6). O teste de milha foi utilizado, de acordo com o index proposto por Kline et al.(9), e os resultados deste teste foram usados para estimativa do consumo máximo de oxigênio ( $\dot{V}_{2}$ max). A flexibilidade foi analisada através de um teste de sentar e alcançar ${ }^{(10)}$. Para avaliação da presença de obesidade, o peso corporal e a altura foram medidas (balança Filizola). As circunferências da cintura e quadril foram também medidas com uma fita métrica. O peso corporal e a altura foram usados para o cálculo do índice de massa corporal (IMC). Além disso, 3 dobras cutâneas (tríceps, subescapular, e torácica para os homens e tríceps, abdominal e supra-ilíaca para as mulheres) foram medidas para estimativa da porcentagem de gordura corporal (\%G) baseadas no protocolo de Jackson e Pollock ${ }^{(11)}$. Tais medidas foram realizadas por um monitor treinado. Os testes físicos e exames laboratoriais foram re-analisados após 4 meses de treinamento e 24-72 horas após a última sessão de exercício.

\section{Programa de treinamento}

A prescrição de exercício individual foi preparada tendo como base os resultados das avaliações físicas e foram monitoradas por um educador físico. Três vezes por semana, os pacientes eram transportados da Unidade Básica de Saúde Otavio Rasi para o Departamento de Educação Física UNESP, Campus de Bauru, num ônibus gentilmente cedido pela Viação Grande Bauru. Este transporte é parte de uma parceria entre a UNESP e a prefeitura de Bauru. A pressão arterial na posição sentada foi aferida através de um esfigmomanômetro no início de cada sessão. Os pacientes recebiam permissão para começarem a sessão de exercício se ambas as pressões arteriais sistólica (PAS) e diastólica (PAD) estivessem abaixo de $140 \times 90 \mathrm{mmHg}$, respectivamente. Além disso, os pacientes que não houvessem tomado suas medicações antihipertensivas não eram permitidos a se exercitarem naquele dia. As PAS e PAD foram usadas para calcular a pressão arterial média (PAM). O programa de exercício incluía exercícios aeróbios (caminhada) a $40-60 \%$ do $\dot{\mathrm{V}} \mathrm{O}_{2}$ max e exercícios de alongamento três vezes por semana, por 90 minutos de sessão total. A intensidade do exercício foi monitorada pela freqüência cardíaca (FC) antes, durante e após cada sessão de exercício aeróbio. Cada monitor era responsável por 5 pacientes.

\section{Análise estatística}

Os valores foram apresentados como média \pm EPM. As comparações entre os dois momentos (inicial e após treinamento), foram calculados através do teste $T$ de Student pareado. Os valores mensais de pressão arterial (PAS, PAD e PAM) representam a média das medidas diárias. Análise de variância de um caminho (ANOVA), com medidas repetidas, foi utilizada para avaliação do perfil de pressão arterial ao longo do programa de treinamento. Quando necessário o teste de Tukey foi utilizado como post-hoc. Para avaliação da correlação entre valores iniciais e resposta após o exercício, foi utilizado o teste de Pearson. O nível de significância utilizado para todos os testes foi de $p<0,05$. 


\section{RESULTADOS}

Dos 40 pacientes, somente 16 mulheres, cuja freqüência de sessões foi maior do que $80 \%$ foram consideradas para a análise

TABELA 1

Efeitos de um programa de exercícios nas características

físicas e variáveis metabólicas de pacientes hipertensos da Unidade Básica de Saúde Otávio Rasi

\begin{tabular}{|c|c|c|}
\hline Variáveis & Iniciais & Finais \\
\hline \multicolumn{3}{|l|}{ Características físicas } \\
\hline$\dot{\mathrm{VO}}_{2} \max \left(\mathrm{ml} \cdot \mathrm{kg}^{-1} \cdot \mathrm{min}\right)$ & $18,01 \pm 2,54$ & $25,86 \pm 2,17^{*}$ \\
\hline Flexibilidade (centímetros) & $25,06 \pm 2,70$ & $27,81 \pm 2,37^{*}$ \\
\hline IMC $\left(K g / m^{2}\right)$ & $31,37 \pm 1,73$ & $31,37 \pm 1,77$ \\
\hline$\%$ Gordura (Percentagem) & $36,67 \pm 1,30$ & $36,58 \pm 1,20$ \\
\hline \multicolumn{3}{|l|}{ Metabolismo lipídico } \\
\hline Níveis de creatina plasmática ( $\mathrm{mg} / \mathrm{dL}$ ) & $0,90 \pm 0,04$ & $0,88 \pm 0,03$ \\
\hline Níveis de glicose plasmática $(\mathrm{mg} / \mathrm{dL})$ & $97,31 \pm 2,75$ & $93,25 \pm 1,90^{*}$ \\
\hline Colesterol total $(\mathrm{mg} / \mathrm{dL})$ & $205,38 \pm 10,15$ & $204,75 \pm 7,59$ \\
\hline LDL - Colesterol (mg/dL) & $119,69 \pm 10,42$ & $114,63 \pm 9,00$ \\
\hline VLDL - Colesterol $(\mathrm{mg} / \mathrm{dL})$ & $28,04 \pm 2,32$ & $28,89 \pm 3,83$ \\
\hline $\mathrm{HDL}$ - Colesterol $(\mathrm{mg} / \mathrm{dL})$ & $54,75 \pm 3,93$ & $60,85 \pm 3,63$ \\
\hline Triglicerídeos $(m g / d L)$ & $143,28 \pm 13,82$ & $138,81 \pm 15,71$ \\
\hline
\end{tabular}

$\dot{\mathrm{V}}_{2}$ max - Consumo máximo de oxigênio; IMC - Índice de massa corporal; \% Gordura - Percentagem de gordura; LDL - lipoproteína de baixa densidade; VLDL - lipoproteína de densidade muito baixa; HDL - lipoproteína de alta densidade. Significância: * vs inicial, p $<0,05$.
(56 \pm 3 anos). Após o treinamento, a eficiência no teste de milha foi significativamente aumentada, expressa por um declínio de 12\% no tempo de caminhada $(17,1$ vs 19,4 minutos ao início do programa, $p<0,05)$. A tabela 1 resume os efeitos de quatro meses de um programa de exercício nas características físicas e metabolismo lipídico. Segundo o aproveitamento no teste de caminhada, o $\dot{\mathrm{V}} \mathrm{O}_{2}$ aumentou em $42 \%$ (tabela 1 ).

Apesar do IMC e \% de gordura não terem se modificado, o programa de exercício foi eficiente para aumentar flexibilidade nesses pacientes $(+11 \%$, tabela $1, p<0,05)$. Ao início de programa de exercício de alongamento, $34 \%$ dos pacientes foram incluídos na variação de flexibilidade normal para a idade $(15 \mathrm{~cm})$, mas após a intervenção, esta porcentagem aumentou até 50\%. Quatro meses de treinamento físico reduziu significativamente o conteúdo de glicose plasmática, como mostrado na tabela 1. Apesar do treinamento não ter modificado significativamente o perfil metabólico do grupo (tabela 1), um fator importante do treinamento físico foi observado quando os valores iniciais individuais foram correlacionados com a resposta após treinamento (figura 1). Como mostrado na figura 1, correlações significativas entre valores iniciais de CT, HDL-C e LDL-C e suas respostas após um programa de exercícios foram vistas. A tabela 2 mostra os valores médios de PAS, PAD e PAM ao longo de 4 meses de treinamento. Cada valor representa uma média de 12 sessões mensais. O exercício aeróbio diminuiu cerca de $8 \mathrm{mmHg}$ de PAS $(p<0,05)$ e $3 \mathrm{mmHg}$ de

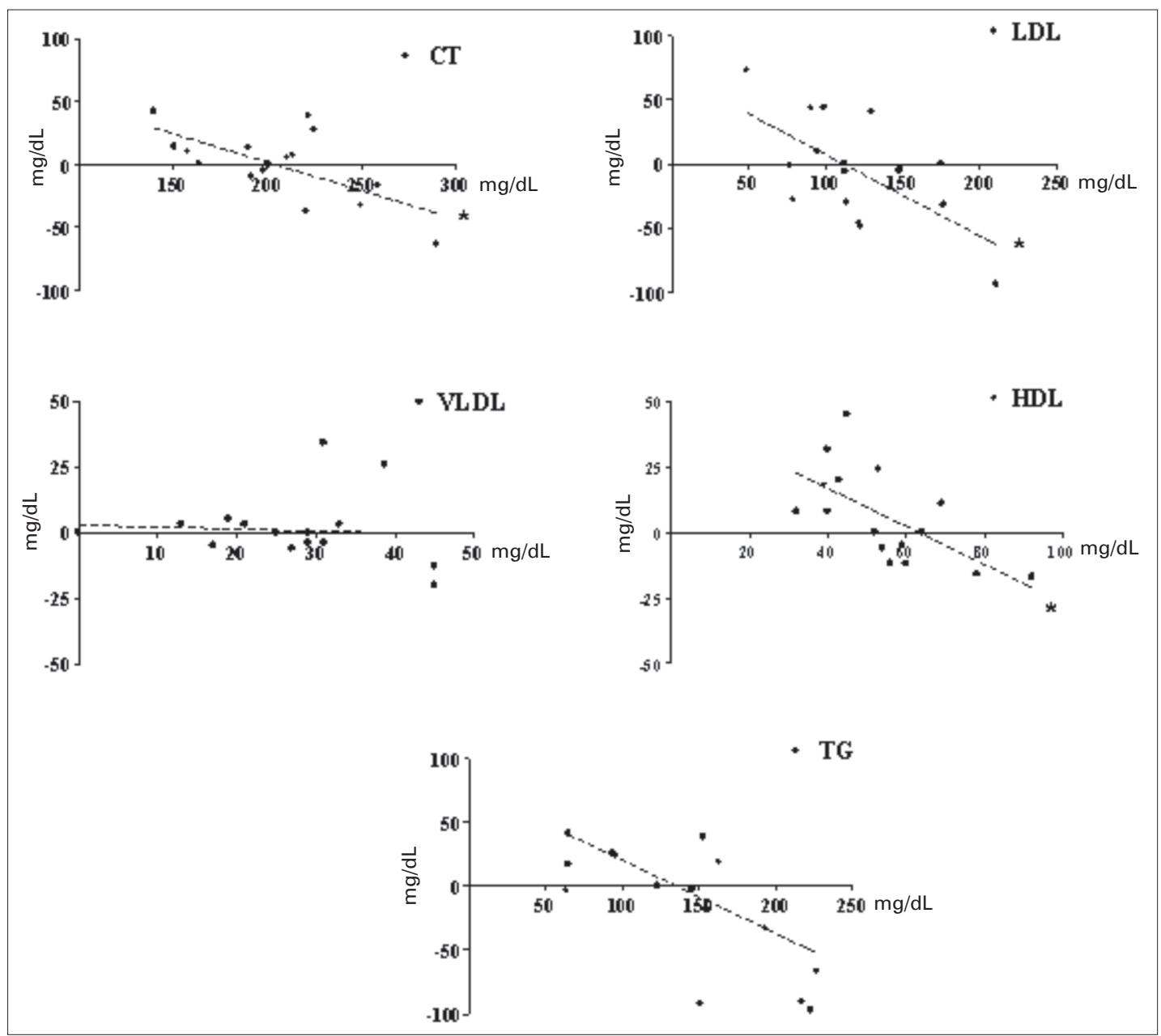

Figura 1 - Correlação entre valores iniciais e resposta após 4 meses de treinamento nas variáveis metabólicas: Colesterol total (CT, $\mathrm{mg} / \mathrm{dL}, y=-0,4530 x \pm 92,4, r=0,6636$; painel A); lipoproteína de baixa densidade (LDL-C, $m g / d L, y=-0,6355 x \pm 71 ; r=0,6301$; painel B); lipoproteína de densidade muito baixa (VLDL-C, $m g / d L, y=-$ $0,07443 x \pm 2,82 ; r=0,661$; painel C); lipoproteína de alta densidade (HDL-C, $m g / d L, y=-0,7303 x \pm 46,08, r=$ 0,6371; painel D), triglicerídeos (TG, $m g / d L, y=-0,2845 x \pm 36,3, r=0,2372$, painel E) e conteúdo plasmático de glicose ( $\mathrm{mg} / \mathrm{dL}, y=-0,4082 x \pm 35,66, r=0,7582$; painel F) em pacientes hipertensos $(n=16)$. Significância: ${ }^{*} p$ $<0,05$. 
ambas PAD e PAM. A tabela 2 mostra que após a terceira semana de treinamento a PAS reduziu-se significativamente. Para se analisar a resposta individual, um teste de correlação foi também aplicado nestas variáveis. Como observado na figura 2, os efeitos do exercício foram mais expressivos em pacientes que apresentaram níveis de pressão altos no início do treinamento.

\section{TABELA 2}

Pressão arterial sistólica de repouso, pressão arterial diastólica e pressão arterial média durante o programa de exercício

\begin{tabular}{cccc} 
Mês & PAS & PAD & PAM \\
1 & $133 \pm 3$ & $83 \pm 2$ & $98 \pm 2$ \\
2 & $129 \pm 1$ & $83 \pm 1$ & $98 \pm 1$ \\
3 & $126 \pm 2^{*}$ & $80 \pm 1$ & $95 \pm 1$ \\
4 & $125 \pm 2^{*}$ & $80 \pm 2$ & $95 \pm 2$ \\
\hline
\end{tabular}

PAS = pressão arterial sistólica; $P A D$ = pressão arterial diastólica; PAM = pressão arterial média Significância: * vs primeiro mês, $p<0,05$.

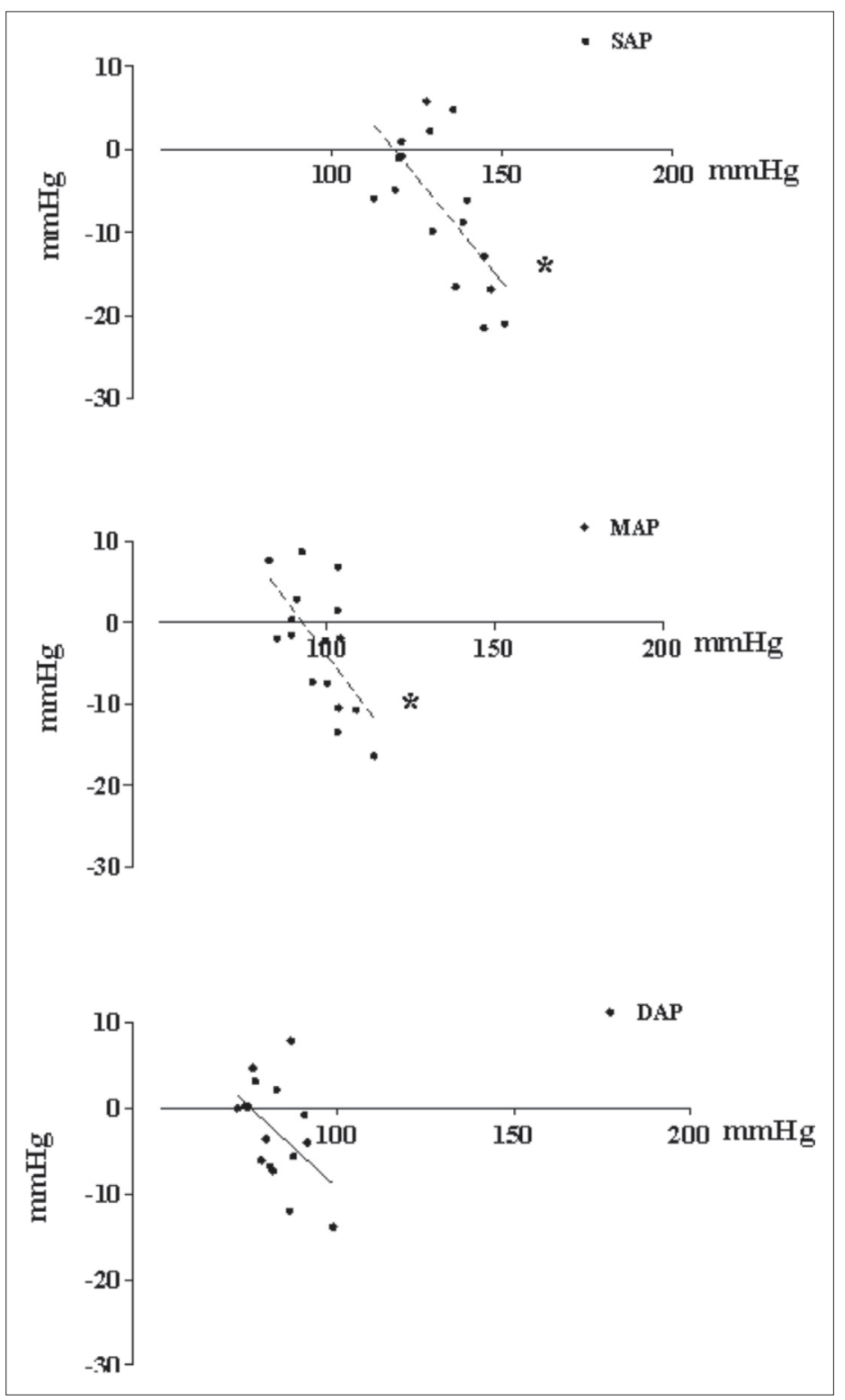

Figura 2 - Correlação entre valores iniciais e resposta após 4 meses de treinamento na pressão arterial sistólica (PAS, $m m H g, y=-0,5064 x \pm$ $59,94, r=0,6674$; painel de cima), pressão arterial média (PAM, $\mathrm{mmHg}, y$ $=-0,5512 x \pm 51,03, r=6372$; painel do meio) e pressão arterial diastólica (PAD, $m m H g, y=-0,3800 \pm 28,64 ; r=0,4771$; painel de baixo) em pacientes hipertensos $(n=16)$. Significância: ${ }^{*} p<0,05$.

\section{DISCUSSÃO}

O resultado principal do presente estudo foi o de que o "Projeto Hipertensão", um programa de condicionamento físico individualizado, criado pelo Departamento de Educação Física da UNESP, Bauru, pode ser introduzido na Unidade Básica de Saúde - Otavio Rasi, para atender pacientes hipertensos. Além disso, quatro meses de exercícios físicos aeróbios e de alongamento, associados a um tratamento farmacológico, foram eficientes para melhorar a capacidade cardiorrespiratória e flexibilidade além de reduzir a pressão arterial em indivíduos hipertensos.

O exercício físico regular ou reabilitação cardíaca utilizando grandes grupos musculares como caminhar, pedalar, correr ou nadar, produz um ajuste cardiovascular que aumenta a capacidade de se exercitar, resistência e força muscular esquelética. Na verdade, ela é considerada uma das principais estratégias em saúde pública, contribuindo para reduzir a morbidade e mortalidade. Tais reduções podem ser mediadas via efeitos indiretos do exercício através da melhora dos fatores de risco como hipertensão, obesidade,

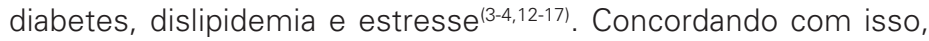
Bonow ${ }^{(18)}$ também apontou que sujeitos que mantêm peso corporal desejável, uma alimentação saudável, exercícios regulares, evitam o fumo e ingerem álcool com moderação; reduzem seu risco de doença cardiovascular em 84\%. Apesar de vários artigos terem mostrado melhora dos fatores de risco cardíaco primário com exercício, o efeito de um programa de exercício regular na qualidade de vida relacionada com saúde continua obscuro.

A hipertensão é um fator de risco importante para doença cardiovascular com maior impacto na morbidade e mortalidade(4). Ela se caracteriza por várias disfunções músculo-esqueléticas, incluindo redução da densidade vascular, a qual pode contribuir para a redução da capacidade aeróbia(19-21). Por outro lado, o treinamento físico aeróbio promove vários ajustes no sistema muscular e cardiovascular. Alguns desses ajustes incluem aumentos na atividade das enzimas oxidativas, assim como no número e tamanho das mitocôndrias. O aumento da rede capilar tem um papel importante na melhora da capacidade aeróbia: facilita o transporte e extração muscular de oxigênio, contribuindo assim para um maior consumo de oxigênio máximo e desempenho físico ${ }^{(22)}$. De acordo com isto, o $\dot{V O}_{2}$ tem sido usado como um marcador de eficiência do treinamento físico. Os resultados do "Projeto Hipertensão" demonstraram que quatro meses de treinamento físico regular são suficientes para melhorar a capacidade aeróbia de indivíduos hipertensos através do aumento do $\mathrm{V}_{2}$ max em cerca de $42 \%$.

Um outro resultado importante do presente estudo é o de que quatro meses de exercício regular foram eficientes para reduzir a PAS de pacientes hipertensos. Esta redução foi mais expressiva e significante após o terceiro mês e não foi associada com a diminuição na PAM e PAD, o que sugere que um período mais longo de exercício poderia alterar efetivamente a pressão arterial. É importante ressaltar que os níveis de pressão arterial não foram muito altos no início do programa de exercícios, uma vez que os pacientes estavam sob tratamento farmacológico. Provavelmente, o resultado principal desta intervenção tenha sido uma associação de fatos que ocorreu com os pacientes, incluindo redução na PA e subseqüente redução no custo com medicação, exames e consultas médicas (dados não apresentados nesta comunicação).

Vários mecanismos podem contribuir para o declínio da pressão sanguínea após a intervenção física. Tem sido mostrado que alterações na pressão sanguínea induzidas por exercícios crônicos são baseados em respostas anatômicas e/ou funcionais, tais como redução de resistência vascular ${ }^{(23)}$, débito cardíaco ${ }^{(24)}$, resistência à insulina(25-27) e atividade simpática(28). O exercício crônico também tem sido associado com o aumento de capilares ${ }^{(22,29-30)} \mathrm{O}$ qual também ajuda a melhorar a capacidade física. 
A melhor recomendação de exercício acerca de quantidade, tipo e intensidade ainda é controversa. O Colégio Americano de Ciências do Esporte ${ }^{(1)}$ recentemente estabeleceu que a maioria dos exercícios recomendados para pacientes hipertensos deveria ser pelo menos 30 minutos de exercícios aeróbios, o máximo de vezes semanais, com intensidade moderada (40-60\% $\left.\dot{\mathrm{VO}}_{2}-\mathrm{R}\right)$. Ao contrário, Ishikawa-Takata et al.(15) demonstraram que oito semanas de exercício aeróbio ( $50 \%$ do $\dot{\mathrm{V}} \mathrm{O}_{2}$ estimado) foram eficientes para reduzir a PA e que 61-90 minutos semanais de exercícios foi meIhor que 30-60 minutos por semana em 207 sujeitos não-tratados com hipertensão essencial. Os autores também sugeriram que futuros aumentos no volume de exercício não melhoraram os resultados. Os resultados do presente estudo mostraram que 135180 minutos/semana (45 a 60 minutos/sessão), foram eficientes para reduzir significativamente a pressão arterial de pacientes hipertensos tratados, o que corrobora com os resultados mostrados por Campane e Gonçalves ${ }^{(2)}$.

Sabe-se que baixo HDL-C associado a alto CT plasmático e LDL-C estão relacionados com alto risco de DCV. Por outro lado, há um grande número de evidências experimentais sugerindo que aumentos no HDL-C e/ou suas apolipoproteínas promovem um efeito protetor importante variando desde prevenção até estabilização e regressão de aterosclerose, independente dos níveis de colesterol(16,31-33). O HDL-C é considerado um mediador importante de transporte de colesterol reverso, um processo que envolve a transferência e assimilação de colesterol livre dos tecidos periféricos, como por exemplo, a parede arterial, com subseqüente envio para o fígado(34). Tais observações confirmam a hipótese de que o treinamento físico, através do aumento o HDLC plasmático e diminuição dos níveis de CT e LDL-C, contribui para a redução do risco de DCV(16,35-36). Shah et al. (32) propuseram em seu artigo que cada $1 \mathrm{mg} / \mathrm{dL}$ de aumento no HDL-C é associado com 2\%-3\% de risco mais baixo de doença coronariana. De acordo com tal fato, nossos dados sugerem que os pacientes hipertensos provavelmente diminuíram cerca de 18\% do risco de doença coronariana, uma vez que os níveis de HDL-C do grupo (tabela 1) aumentaram $6 \mathrm{mg} / \mathrm{dL}$ após o programa de exercício físico. Embora a média do grupo não tenha mostrado um aumento significativo $(11 \%, p>0,05)$ nos níveis de HDL-C, uma resposta individual de treinamento físico foi demonstrado com uma correlação significativa entre valores de base de HLD-C e alterações de HDL-C pós-exercício, o que significa que pacientes que apresentaram níveis de HDL-C menores ao início do programa obtiveram maiores benefícios do treinamento físico ( $r=0,6373$, $P<0,05)$. Já que a população era tão heterogênea, com estilos de vida, alimentação, peso e condições de vida diferentes, não foi surpresa que não tenha sido encontrada nenhuma significância quando os grupos foram comparados. Na verdade, o essencial a ser considerado é que indivíduos que tinham valores alterados usufruíram dos maiores benefícios do exercício. O resultado deste estudo está de acordo com aqueles apresentados por Zmuda et al. (37) os quais mostraram uma melhora de HDL-C induzida pelo exercício em sujeitos com níveis baixos de HDL-C. É importante ressaltar que a melhora nos níveis de HDL-C encontrados neste estudo $(+11 \%)$ foi parecida com a apresentada por outros autores usando somente tratamento farmacológico(38) e os pacientes não estavam fazendo uso de medicação para ajustes de perfil lipídico. Couillard et al. ${ }^{(35)}$ mostraram que o exercício se mostra eficiente no aumento de HDL-C se o estímulo do treinamento físico é suficiente. Além disso, vários estudos sugeriram que o efeito de aumento do HDL pelo treinamento físico poderia ser amplamente explicado através da perda concomitante de gordura corporal(39). Nosso estudo não tinha recomendação nutricional e isso provavelmente tenha contribuído para as modificações não-significativas no perfil lipídico. Os níveis de CT e LDL-C tiveram o mesmo padrão, mas em direções opostas. Os pacientes que tiveram CT basal mais altos ou cujos níveis de
LDL-C tiveram mudanças mais significativas apresentaram alterações mais expressivas após a intervenção.

Taylor et al. ${ }^{(33)}$ recentemente publicaram uma metanálise de 48 estudos com um total de 8.940 pacientes submetidos a um programa de reabilitação cardíaca de 3-15 meses. Esta revisão confirmou os benefícios da reabilitação cardíaca baseada em exercícios em mortalidade de todas as causas, o qual foi associado com reduções de CT, TG e PAS. Não foram encontradas diferenças nos níveis de colesterol de lipoproteínas de alta e baixa densidade dos pacientes. Os autores sugerem que provavelmente seria necessário mais tempo para a melhora destas variáveis. Estes resultados representam um sinal encorajador para que o número de programas de reabilitação baseados em exercícios como o do nosso estudo seja aumentado.

Um outro risco comumente presente em adultos é a lesão músculo-esquelética, a qual pode ser exacerbada por baixa flexibilidade. O processo degenerativo advindo do envelhecimento contribui para a diminuição da amplitude de movimento e limita a mobilidade. O risco de lesão aumenta com a obesidade, volume e intensidade do exercício; porém, quando indivíduos com alto risco estão excluídos, as lesões são raras no treinamento físico supervisionado em adultos idosos ${ }^{(16)}$. Nesta investigação, 34\% dos pacientes foram incluídos na faixa de flexibilidade normal para a idade na avaliação inicial e, após a intervenção com exercícios de alongamento e flexibilidade, esta taxa aumentou para $50 \%$. Os exercícios de alongamento e flexibilidade recomendados foram feitos três vezes por semana por 30 minutos em cada sessão, o que está de acordo com as recomendações da $\operatorname{ACSM}^{(40)}$. Heyward(41) sugeriu uma combinação de exercícios de alongamento e força para os músculos abdominais e lombares para prevenção de problemas lombares.

Em resumo, os resultados do presente estudo mostraram que o Projeto Hipertensão, um programa baseado em exercício desenvolvido pelo departamento de Educação Física da UNESP, Bauru, foi introduzido com sucesso e confirmaram os benefícios do exercício na diminuição da PA, aumento do condicionamento físico e flexibilidade de pacientes hipertensos da Unidade Básica de Saúde Otavio Rasi. Embora uma correlação significativa entre níveis basais de parâmetros metabólicos (HDL-C, CT e LDL-C) e suas respectivas modificações pós-exercício tenha sido encontrada, um programa de exercícios físicos mais prolongado, associado a um controle nutricional, pode ser necessário para a melhora das características físicas e perfil lipídico. A redução expressiva de PA em sujeitos hipertensos sugere que esta intervenção baseada em exercício deve ser enfatizada em outros centros de saúde que assistam populações de baixa renda.

\section{AGRADECIMENTOS}

Os autores agradecem à enfermeira Anair dos Santos Freitas por sua assistência profissional com os pacientes. Livia Maria Castro Rolim foi aluna financiada pela bolsa de Iniciação Científica da Fundação de Amparo à Pesquisa do Estado de São Paulo (FAPESP). Fernando Cadamuro Silva foi aluno financiado por bolsa de Iniciação Científica do Conselho Nacional de Desenvolvimento Científico e Tecnológico (PIBIC/CNPq).

Todos os autores declararam não haver qualquer potencial conflito de interesses referente a este artigo.

\section{REFERÊNCIAS}

1. Pescatello LS, Franklin BA, Fagard R, Farquhar WB, Kelley GA, Ray CA. Exercise and hypertension. Med Sci Sports Exerc. 2004;36 (3):533-53.

2. Campane RZ, Gonçalves A. Atividade física no controle da hipertensão arterial. Rev Bras Med. 2002;59(8):561-7.

3. Fang J, Wylie-Rosett J, Alderman MH. Exercise and cardiovascular outcomes by hypertensive status: NHANES 1 epidemiologic follow-up study. Am J Hypertens. 2005;18:751-8. 
4. Chobanian, et al. The seventh report of the Joint National Committee on prevention, detection, evaluation and treatment of high blood pressure. JAMA. 2003; 289:2560-72.

5. Brasil. Ministério da Saúde. Sistema Único de Saúde. http://tabnet.datasus.gov.br. 20/07/2005.

6. V Diretrizes Brasileiras de Hipertensão Arterial. Hipertensão. Sociedade Brasileira de Hipertensão, 2006

7. Chruch TS, et al. Usefulness of cardiorespiratory fitness as a predictor of allcause and cardiovascular disease mortality in men with systemic hypertension. Am J Cardiol. 2001;88:651-6.

8. Mokdad AH, Marks JS, Stroup DF, Geberding JL. Actual causes of death in the United States, 2000. JAMA. 2004;291:1238-45

9. Kline GM, Porcari JP, Hintermeister R, Freedson OS, Ward A, McCarron RF, et al. Estimation of $\dot{\mathrm{V}}_{2}$ max. from a 1-mile track walk, gender, age and body weight. Med Sci Sports Exerc. 1987;19:253-359

10. Guedes DP, Guedes JERP. Crescimento, composição corporal e desempenho motor. São Paulo: CLR-Baliero, 1997.

11. Jackson AS, Pollock ML. Practical assessment of body composition. Phys Sports Med. 1985;13:76-90

12. Tsai JC, Liu JC, Kao CC, Tomlinson B, Kao PF, Chen JW, et al. Beneficial effects on blood pressure and lipid profile of programmed exercise training in subjects with white coat hypertension. Am J Hypertens. 2002 Jun;15(6):571-6.

13. Andrade JP, Vilas-Boas F, Chagas H, Andrade M. Aspectos epidemiológicos da aderência ao tratamento da hipertensão arterial sistêmica. Arq Bras Cardiol. 2002; 79(4):375-9.

14. Mion D Jr, Pierin AM, Guimaraes A. Treatment of hypertension - answers of Brazilian physicians to a survey. Rev Assoc Med Bras. 2001;47(3):249-54.

15. Ishikawa-Takata KI, Ohta T, Tanaka H. How much exercise is required to reduce blood pressure in essential hypertensives: a dose response study. Am J Hypertens. 2003;6(8):629-33.

16. Thompson PD, Buchner D, Pina IL, Balady GJ, Williams MA, Marcus BH, et al American Heart Association Council on Clinical Cardiology Subcommittee on Exercise, Rehabilitation, and Prevention; American Heart Association Council on Nutrition, Physical Activity, and Metabolism Subcommittee on Physical Activity. Exercise and physical activity in the prevention and treatment of atherosclerotic cardiovascular disease: a statement from the Council on Clinical Cardiology (Subcommittee on Exercise, Rehabilitation, and Prevention) and the Council on Nutrition, Physical Activity, and Metabolism (Subcommittee on Physical Activity). Circulation. 2003;107(24):3109-16

17. Forjaz CLM, Santanella DF, Souza MO. Atividade física para pessoa hipertensa In: Pierin AMG. Hipertensão arterial: uma proposta para o cuidar. São Paulo: Manole, 2004.

18. Bonow RO. Primary prevention of cardiovascular disease: a call to action. Circulation. 2002;106:3140-1.

19. Amaral SL, Zorn TMT, Michelini LC. Exercise training normalizes wall-to-lumen ratio of the gracilis muscle arterioles and reduces pressure in spontaneously hypertensive rats. J Hypertens. 2000;18:1563-72.

20. Hagberg JM, Park JJ, Brown MD. The role of exercise training in the treatment of hypertension: an update. Sports Med. 2000;30:193-206.

21. Tipton CM. Exercise training for treatment of hypertension: a review. Clin J Sports Med. 1999:9:104

22. Richardson RS, Wagner H, Mudaliar SRD, Saucedo E, Henry R, Wagner PD. Exercise adaptation attenuates VEGF gene expression in human skeletal muscle. Am J Physiol Heart Circ Physiol. 2000;279:H772-H778.
23. Nelson L, Jennings GL, Esler MD, Korner PI. The effect of changing levels of physical activity on blood pressure and hemodynamics in patients with essential hypertension. Lancet. 1986;2:474-6.

24. Veras-Silva AS, Mattos KC, Gava NS, Brum PC, Negrão CE, Krieger EM. Lowintensity exercise training decreases cardiac output and hypertension in spontaneously hypertensive rats. Am J Physiol. 1997;273:H2627-H2631.

25. Forjaz CL, Tinucci T, Oliveira Alonso D, Negrão CE. Exercício físico e diabete. Rev Soc Cardiol Estado de São Paulo. 1998;8(5):981-7.

26. Gautier JF, Mauvais-Jarvis F. Physical exercise and insulin sensitivity. Diabetes Metab. 2001;27:255-60.

27. Rhéaume C, Waib PH, Lacourcière $Y$, Nedeau A, Cléroux J. Effects of mild exercise on insulin sensitivity in hypertensive subjects. Hypertension. 2002;39:98995

28. Roveda F, Middlekauff HR, Rondon MU, Reis SF, Souza M, Nastari L, et al. The effects of exercise training on sympathetic neural activation in advanced heart failure: a randomized controlled trial. J Am Coll Cardiol. 2003;42(5):854-60

29. Amaral SL, Papanek PE, Greene A. Angiotensin II and VEGF are involved in angiogenesis induced by short-term exercise training. Am J Physiol Heart Circ Physiol. 2001;281:H1163-H1169.

30. Gustafsson T, Kraus WE. Exercise-induced angiogenesis-related growth and transcription factors in skeletal muscle, and their modification in muscle pathology. Frontiers in Bioscience. 2001;6:D75-D89.

31. O'Connell BJ, Genest J Jr. High-density lipoproteins and endothelial function. Circulation. 2001 Oct 16;104(16):1978-83.

32. Shah PK, Kaul S, Nilsson J, Cercek B. Explointig the vascular protective effects of high-density lipoprotein and its apolipoproteins. Circulation. 2001;104:2376-83.

33. Taylor RS, Brown A, Ebrahim S, Jolliffe J, Noorani H, Rees K, et al. Exercisebased rehabilitation for patients with coronary heart disease. Am J Med. 2004; 116:682-92.

34. Spady DK. Reverse cholesterol transport and atherosclerosis regression. Circulation. 1999;100(6):576-8

35. Couillard C, Despres JP, Lamarche B, et al. Effects of endurance exercise training on plasma HDL cholesterol levels depend on level of triglycerides: evidence from men of the Health, Risk Factors, Exercise Training and Genetics (HERITAGE) Family Study. Arterioscler Thromb Vasc Biol. 2001;21:1226-32.

36. Leon AS, Rice T, Mandel S, et al. Blood lipid response to 20 weeks of supervised exercise in a large biracial population: the HERITAGE Family Study. Metabolism. 2000;49:513-20.

37. Zmuda JM, Yurgalevitch SM, Flynn MM, Bausserman LL, Saratelli A, SpannausMartin DJ, et al. Exercise training has little effect on HDL levels and metabolism in men with initially low HDL cholesterol. Atherosclerosis. 1998;137:215-21.

38. Huttunen JK, Manninen V, Manttari M, et al. The Helsinki Heart Study: central findings and clinical implications. Ann Med. 2001;23:155-9.

39. Fagard $\mathrm{RH}$. Physical activity in prevention and treatment of hypertension in the obese. Med Sci Sports and Exerc. 1999;31(11)Suppl 1:S624-S630.

40. Cress M Elaine, Buchner David M, Prohaska, Thomas. Physical Activity Programs and Behavior Counseling in Older Adult Populations. The American College Sports Medicine recommendation. Med Sci Sports Exerc. 2004;36(11):1997-2003.

41. Heyward VH. Advanced fitness assessment \& exercise prescription. Human Kinetics Europe, 1998 